\title{
Carnets
}

Revue électronique d'études françaises de l'APEF

Deuxième série - 9 | 2017

Reconnaissances et légitimité en français

\section{Ce que nous devons à l'enseignement}

De la littérature (française)

\section{Sidi Omar Azeroual}

\section{(2) OpenEdition}

Journals

Édition électronique

URL : http://journals.openedition.org/carnets/2131

DOI : 10.4000/carnets.2131

ISSN : 1646-7698

Éditeur

APEF

Référence électronique

Sidi Omar Azeroual, «Ce que nous devons à l'enseignement », Carnets [En ligne], Deuxième série -

9| 2017, mis en ligne le 31 janvier 2017, consulté le 01 mai 2019. URL : http://

journals.openedition.org/carnets/2131; DOI : 10.4000/carnets.2131

Ce document a été généré automatiquement le 1 mai 2019.

\section{(c) (i) \&}

Carnets est mis à disposition selon les termes de la licence Creative Commons - Atribution - Pas

d'utilisation commerciale 4.0 International. 


\section{Ce que nous devons à l'enseignement}

De la littérature (française)

\section{Sidi Omar Azeroual}

\section{NOTE DE L'AUTEUR}

Cet article est axé sur la légitimité de l'enseignement de la littérature. S'il réfère explicitement à la littérature française, que je mets délibérément entre parenthèses, c'est uniquement par contrainte culturelle : l'enseignement dont j'ai disposé est étroitement lié à la culture française. Toute autre littérature - dite étrangère -, hormis la littérature arabo-musulmane qui cadre mon identité intellectuelle, je l'ai rencontrée dans la langue française. D'une manière ou d'une autre, dans mon imaginaire, cette autre littérature fait partie intégrante de la langue et de la littérature française.

«Oui, il y a des livres qui m’ont marqué à jamais,
qui m'ont transformé : Baudelaire, Rilke, Pascal,
Spinoza, Hume, Montaigne, Aristote, ... Aussi
Proust et Céline... Et puis, avant tous ceux-là, Les
Thibault, de Martin du Gard, et Les Nourritures
terrestres, de Gide »(Comte-Sponville, $2000: 121)$.

\section{Langue française et discours politique}

\section{I- 1- Le cas du Maroc}

1 La question de la langue demeure épineuse au Maroc où l'on n'est pas tous d'accord sur ce qu'est une langue officielle. Est-ce l'arabe, langue du sacré, ou l'amazigh, dialecte des origines? Est-ce le français enraciné et entretenu par les anciens colons ou l'espagnol qui a envahi le nord du pays? Il faudrait dans ce cas procéder par hiérarchisation des 
langues. Si l'amazigh est considéré comme un dialecte à valeur culturelle et l'arabe comme une pseudo langue officielle, le français s'impose socialement et a la mainmise sur la paperasse administrative. De même, il est la langue des prospectus, des ordonnances médicales, des projets de loi, des communiqués ... etc.

2 Toutefois, le pouvoir politique actuel, monopolisé par le parti à référence islamique, propose, au nom de Monsieur Lahcen Daoudi, ministre de l'enseignement supérieur, de penser à l'anglais comme un outil pragmatique incontestable. Le ministre, cité par Amal Galla, a explicitement affirmé qu'au Maroc, «l'on ne maîtrise ni l'arabe ni le français, donc il faudrait surtout maîtriser l'anglais, et étudier alors en arabe, et ce pour mieux profiter des références scientifiques qui sont surtout en anglais » (Galla, 2014). Il ajoute dans ce sens que le pays a besoin des sciences exactes plus que des sciences humaines. Tandis qu'une seule compagnie aéroportuaire, selon les dires du ministre, demande plus de 700 ingénieurs techniciens, des centaines d'autres établissements classiques (facultés, écoles privées...) congédient des salariées et des fonctionnaires dont le péché est de ne pas avoir été assez malin pour se former en d'autres disciplines plus rentables.

3 Les Etudes Françaises (langue et littérature) est l'une des filières importantes à l'université marocaine. Désormais, selon le ministre de l'enseignement supérieur marocain, ce département est inutile. Or, au lieu de parler en termes de table rase, il faudrait peut-être prévoir une restructuration de la représentation sociale où chaque individu cherche à ensevelir sa déception due au décalage entre un nouveau contexte intellectuel et un idéal fané. Le discours politique est responsable des répercussions de cette déception.

4 La question de la légitimité de la langue française que, plus ou moins, tous les marocains comprennent (en dépit du degré de la maîtrise de la grammaire et de la syntaxe) nécessite un engagement dans un débat sur la nature du projet social adéquat au pays. Ce débat doit exiger de déterminer, parallèlement, la nature de la langue qui saurait être conforme à la représentation des citoyens.

C'est en plaçant la problématique sur ces bases que l'on pourrait finalement décider de l'importance de Balzac, de Saussure, de Diderot, de Duras et de bien d'autres figures d'une culture qui a toujours contribué à ce qu'est aujourd'hui cet univers francophone ouvert sur le monde. Il serait donc aberrant d'entendre un responsable dire que si l'on s'éloigne des théories des économistes (surtout anglophones), on s'égare du droit chemin, que supprimer Victor Hugo de l'histoire ne nuira pas à l'imaginaire humain, que la révolution française n'a pas été inaugurée par les écrits des philosophes français, mais par la faim du peuple.

6 À quoi servirait donc la littérature si elle ne prétend pas faire partie d'une université qui « entretient des relations complexes avec la société, sous la forme de demandes et d'offres de plus en plus déréglées » (de Certeau, 1993: 113) ? La réponse de Belinda Cannone s'arrête judicieusement sur ce qui constitue la richesse de la création littéraire :

Les romanciers ont toujours fondé la légitimité de leur entreprise sur cette conviction qu'une chose réelle n'a pas plus d'importance qu'une chose pensée. C'est celui-là qui, pour la première fois, donne aux possibilités nouvelles leur sens et leur destination, c'est celui-là qui les éveille' (L'Homme sans qualités). Le romancier comme éveilleur des possibles (Cannone, 2012 : 87-88).

7 Il est à noter que la délégitimation de la littérature française ne date pas d'aujourd'hui puisque déjà Nicolas Sarkozy, ancien président français, censé défendre la culture française, critique en 2006 comme en 2008 La Princesse de Clèves ${ }^{1}$. Si l'on voudrait donc 
continuer à enseigner la langue et la littérature françaises dans un pays francophone, sur quel type de reconnaissance sociale faudrait-il bâtir les critères de l'apprentissage ? Parce qu'on ne va pas nécessairement à l'école pour devenir, des années après, des chefs d'entreprises, parce que l'école enseigne d'abord ce qu'est un être humain, la réponse à cette question est fondamentale.

\section{I- 2- Le cas de l'Europe non francophone}

8 À la fin du XXIII ${ }^{\text {ème }}$ colloque international de l'APFUE ${ }^{2}$, le sujet d'une table ronde tournait autour de la situation et du statut du français en Espagne. Ce débat microcosmique est une illustration parfaite du combat que mènent l'ensemble des associations issues de sociétés non-francophones et qui luttent pour un potentiel rayonnement du français parmi les différentes couches sociales. La consultation d'une série d'articles spécialisés sur le même sujet permet d'aboutir aux mêmes conclusions pessimistes dont souffre le corps enseignant espagnol, portugais, roumain, italien, autrichien, ... etc.

Quatre points indispensables devraient être immédiatement pris en considération si l'on voudrait contribuer à la légitimation du français et de la culture française.

1-Tout d'abord, il est recommandé de questionner la valeur du français d'un point de vue sociologique : est-il une langue utile, donc à concevoir comme seconde langue privilégiée, ou une langue de prestige, donc simple langue étrangère à revaloriser selon des objectifs précis?

2-Dans cet ordre d'idées, il faudrait réduire l'impact de l'hétérogénéité linguistique sur la confusion du choix de la langue qu'on voudrait apprendre. C'est le cas de la politique linguistique au Portugal où la situation du français reste problématique malgré les efforts des agents associatifs.

3-De même, il est nécessaire de prévoir une formation des enseignants du français. Le cas des professeurs d'espagnol qui prennent maladroitement en charge l'enseignement du français est un exemple parmi tant d'autres.

4-La mise à niveau de la qualité de l'enseignement du français nous incite également à repenser le rôle de la littérature dans l'ancrage de la langue française en vue de motiver des apprenants souvent indifférents.

10 Ces efforts ne sauraient aboutir à leur finalité si l'on ne prend pas conscience que le changement du statut du français, langue minoritaire au sein des pays non-francophones, exige une modification radicale des soubassements pédagogiques de tout un état. Citons encore le cas de l'Espagne qui a opté pour le remplacement de certaines matières (comme la musique) par des langues étrangères fragilisées. Un véritable plan d'urgence à la fois d'ordre politique, sociologique, pédagogique et culturel est plus que nécessaire.

\section{II- Le nouvel idéal de l'enseignement universitaire}

\section{II- 1- Invasion technologique}

Le changement des notions est favorisé par le changement de la vision du monde, ce qui se répercute sur l'intérêt octroyé à l'idée de l'enseignement. Si, auparavant, l'enseignement de l'informatique était secondaire parce qu'il se réduisait à quelques principes de traitement de texte que les étudiants, en général, sont censés maîtriser, aujourd'hui, des filières d'informatiques accueillent un assez bon nombre de doctorants 
et d'étudiants avides de programmation et de savoir purement scientifique. En linguistique, l'intérêt donné au cognitivisme est étroitement lié au développement technologique du traitement $d u$ fonctionnement du cerveau. La linguistique, après des années de familiarité avec la psychologie et la sociologie, est désormais inséparable de la biologie. L'ère technologique, qui succède à un modernisme attaché au principe - encore actuel - de la mondialisation, exige une extraction de tout ce qui ne répond pas aux critères d'un marché nourri par un capitalisme financier et un impérialisme culturel.

Or, l'université n'est pas un espace d'ambition économique, mais d'émerveillement philosophique devant les grandes idées susceptibles d'approfondir chez l'étudiant une conscience politique vis-à-vis des idéologies dominantes. L'enjeu d'une formation n'est pas nécessairement d'éveiller une conscience malheureuse qui déboucherait sur un engagement politique. Il suffit d'éveiller chez l'étudiant le désir d'être libre penseur, lecteur averti du quotidien, bricoleur face aux conflits culturels ou dénonciateur des faux-fuyants. En l'absence d'une telle mission, apparaît aussi bien chez l'enseignant que chez l'étudiant "un sentiment d'insécurité » (de Certeau, 1993: 112). Ce qui enraidit l'opacité de ce paysage brumeux, c'est l'émergence de nouveaux lieux de la culture: "l'usine, les mass media, les techniques, les grandes entreprises» (ibidem). Michel de Certeau résume ainsi cette situation : « L'enseignant flotte à la surface de la culture (...)» ( ibidem).

13 La culture perd du terrain et cède de plus en plus la place aux sciences exactes. De grands noms comme Claude Lévi-Strauss, Marcel Mauss, Marcel Proust, Paul Valéry et André Gide disparaissent insensiblement et sont remplacés par d'autres icônes omniprésentes (Steve Jobs, Mark Zuckerberg, Bill Gates, ...). Il ne reste de Balzac que le nom de la rue, à Tours en France, où se situe sa maison d'enfance, patrimoine destiné à attirer quelques touristes avertis. Balzac relève désormais non plus de la culture proprement dite, mais de la culture générale (quelques bribes superficielles et lacunaires sur l'auteur et le résumé anodin de ses livres). Cette situation nous renseigne sur la mort symbolique de l'histoire des idées et l'entrée solennelle de l'humanité dans l'ère de la sacralisation de l'éphémère, l'actualité évanescente ici et maintenant grâce à une surinformation qui ne demande qu'à être exhibée immédiatement, puis oubliée ou refoulée.

Le progrès technologique se fonde sur une mise en scène ostentatoire de l'imagination purement matérielle ${ }^{3}$. Cependant, sans littérature - et, dans le cas des pays francophones, sans littérature française - il n'y aurait point d'imagination purement abstraite, celle qui, chez l'homo habilis, privilégie l'idée, non l'objet. Ainsi, afin de ne pas succomber aux différentes menaces qui l'entourent, il est fondamental de prendre au sérieux l'enseignement des lettres, c'est-à-dire en tant qu'un réservoir d'idées philosophiques, non de formes uniquement littéraires.

\section{II- 2- Littérature et exigences du marché}

ne faut pas maltraiter la littérature en la soumettant aux nouvelles logiques du marché qui exigent qu'un enseignement doive nécessairement déboucher sur un vrai métier, sur une certaine rentabilité économique de l'individu. Et que peut la littérature devant ces nouveaux métiers qui lui sont étrangers et qui lui ôtent toute chance d'avoir une légitimité sociale.

La littérature garantit le passage d'un état de découverte du monde à une maturité culturelle capable d'initier l'apprenant à la relation sociale. Qu'on ne puisse pas intégrer 
la littérature dans la programmation de ce que l'on appelle les « grandes écoles » ne veut pas dire que, désormais, elle est inutile. D'ailleurs,

(...) comme le souligne une prise au sérieux de son étymologie, l'éducation est une conduite, un accompagnement, qui aboutit à une conversion, une transformation. Elle est en tous cas une intervention. L'existence des différences, et donc de la diversité, induit alors plusieurs types d'attitudes théoriques et pratiques (DrouinHans, 1998 : 201).

De sa part, l'auteur de La Culture au pluriel (de Certeau, 1993: 112) distingue deux tendances :

1- Un courant réaliste, produit scolaire, qui privilégie la valeur d'échange, fondée sur une vision pragmatique visant des finalités économiques (la valeur d'usage, elle, est marginalisée parce qu'elle vise des préoccupations personnelles).

2-Un courant culturel, produit des sciences humaines, destiné aux salariés, aux personnes âgées, aux riches, ... dont l'objectif est l'exploration des pistes de réflexion.

Le changement des noms des établissements de formation contribuerait à l'enracinement de la tendance culturelle de l'éducation. Au lieu de l'université ou de la faculté, on pourrait les appeler « Maison(s) de la culture » (ibidem). La formation aussi devrait subir un changement exhaustif des contenus dispensés. De nos jours, qui voudrait encore lire Morphologie des contes, Du sens de Greimas, Forme et signification de Jean Rousset ou Physiologie de la critique d'Albert Thibaudet?

cadre des études françaises, il est peut-être temps d'arrêter d'enseigner une narratologie qui ne servirait à rien d'un point de vue culturel. La critique littéraire également, les catégories du récit, une histoire des idées et des arts influencée par l'esprit des manuels, ... Quand on enseigne la poésie de Senghor, c'est à l'essence de la négritude et les soubassements d'une identité locale qu'on devrait s'intéresser. De même, enseigner Alain Robbe-Grillet sous l'égide du Nouveau Roman risque de n'intéresser plus personne. Jamais un malentendu n'a touché une science comme il touche aujourd'hui le domaine des sciences humaines. On a vite oublié que les institutions littéraires dispensent une formation intellectuelle, non professionnelle. De cette formation personne ne veut. On surcharge des branches, comme la littérature, d'un fardeau qui ne relève pas de leur nature.

21 La littérature n'a jamais prétendu préparer ses disciples à autre chose qu'à la réflexion. Aujourd'hui, l'accuser de non rentable, c'est la comparer à des disciplines d'une autre nature, catégoriquement différente de celle assignée aux hommes de lettres. Adopter cette comparaison, c'est vouloir délibérément la condamner à mort.

Aristote a beau chercher à expulser les poètes de sa cité idéale! En vain. Imaginons un pays sans poètes, sans romanciers, sans peintres ou sans dramaturges. Imaginons la France sans Baudelaire ou Mallarmé, sans Proust, Lautréamont, Hugo, Lamartine, Racine, ... Elle serait autre chose que la France. Peu importe ce que l'on est quand on manque d'identité culturelle au sein d'une mondialisation autoritaire.

Cette mondialisation qui est d'abord une quête de pouvoir économique à exercer sur le monde refuse de se soumettre à tout ce qui relève de l'éthique humaniste. Celle-ci se fonde sur la culture et la quête de l'échange. Il n'est donc pas étrange de se confronter, surtout après la crise de 2008, à des décisions qui visent la stabilisation économique des sociétés au détriment de l'essence de l'être humain. La vérité littéraire et philosophique n'intéresse plus les institutions académiques qui préfèrent redéfinir certaines notions nécessaires à l'évolution intellectuelle du citoyen. Ainsi, lorsque le Japon aspire à mettre 
fin au règne des sciences humaines, lorsque la France décide d'abandonner le grec et le latin et l'Espagne la musique, les hommes politiques n'ont pas tort d'un point de vue économique. La vérité est sacrifiée au profit d'une culture rentable.

\section{III- Leçons de littérature}

\section{III- 1- Désir et fictions humanistes} conscience de l'authenticité que l'écriture offre généreusement quand on s'identifie à ses formes :

Rêver du livre que l'on est en train d'écrire (...) : je m'étais endormi sur le tapis, au pied de la bibliothèque. J'étais le dernier paragraphe, son ondulation, sa modulation. Surpris, navré, amusé de me re/trouver quand même avec un corps, 
alors que j'étais passé de l'autre côté : dans l'air, entre les phrases (Sollers, 2006 :

9-10).

Ce qui peut paraître ici un simple fantasme de nuit, change de sens quand on le confronte à ce que dit Michel Leiris de son autobiographie L'Âge d'homme : « il s'agissait moins là de ce qu'il est convenu d'appeler "littérature engagée" que d'une littérature dans laquelle j'essayais de m'engager tout entier » (Leiris, 1939 : 15). Devenir paragraphe, c'est dompter l'espace sauvage qui sépare la corporalité et la scripturalité. C'est aussi humaniser une écriture inséparable du désir de s'engager entièrement dans l'acte de la création encore inachevée du monde.

Grâce à la magie de l'écriture, le scribe franchit le seuil de l'arène et devient, malgré lui, à la fois le taureau et le torero. Plus qu'un dernier paragraphe, il se livre à la tentation de tromper sa plume dans son propre sang ${ }^{5}$, dans l'encre amère qui écrit la tragédie de l'être. ๔Edipe, écrivain, crève ses yeux afin d'accéder à la clairvoyance ; son écriture consume ses yeux, non son regard. Aveugle, il voit autre chose que le monde, la vérité, celle qu'Annie Ernaux définit ainsi : « (...) ce qu'on cherche et qui se dérobe sans cesse» (Ernaux, 2011: 30), et qu'on ne peut retrouver que dans la littérature. Ce qui se dérobe promet une réflexion sur les limites, mission que la littérature (française en particulier) nous apprend « (...) depuis cent ans, de Mallarmé à Blanchot (...)» (Barthes, 1981 : 33).

Ainsi, pour que la littérature puisse jouir d'une légitimité institutionnelle et sociale, il faudrait la considérer sous l'angle de ce qu'elle pourrait représenter. Outre sa dimension esthétique, écrire est un acte psychanalytique qui permet l'exploration intime de ce que l'on est véritablement. Il est également un acte sociologique fondé sur l'image que l'écrivain donne de lui-même (voir Leiris, 1939: 14-15). Finalement, elle est un acte philosophique qui pose des questions sur l'homme et l'humanité, y compris sur ceux qui dévalorisent l'enseignement de la littérature.

\section{BIBLIOGRAPHIE}

BARTHES, Roland (1981). Le Grain de la voix. Paris : Seuil.

COMTE-SPONVILLE, André (2000). L'Amour la solitude. Paris : Albin Michel.

CANNONE, Belinda (2012). L'écriture du désir. Paris : Gallimard.

DE CERTEAU, Michel (1993). La Culture au pluriel. Paris : Seuil.

DROUIN-HANS, Anne-Marie (1998). « Diversité ou identité : quel idéal pour l'éducation ? Opposition ou convergences? ", Diversidade e identidade, Actas da $1^{\text {a }}$ Conferência Internacional de Filosofia da Educação [disponible le 20/10/2015] <http://ler.letras.up.pt/uploads/ficheiros/6204.pdf >.

ERNAUX, Annie (2011). L'Ecriture comme un couteau. Entretien avec Frédéric-Yves Jeannet. Paris : Gallimard.

FABRE, Clarisse (2011). « Et Nicolas Sarkozy fit la fortune du roman de Mme de La Fayette » [disponible le 20/10/2015] < http://www.lemonde.fr/cinema/article/2011/03/29/et-nicolassarkozy-fit-la-fortune-du-roman-de-mme-de-la-fayette_1500132_3476.html>. 
GALLA, Amal (2014). « Lahcen Daoudi : 'La langue dont nous avons besoin aujourd'hui est l'anglais et non le français'» [disponible le 20/10/2015] <http://www.lemag.ma/Lahcen-Daoudi-La-languedont-nous-avons-besoin-aujourd-hui-est-l-anglais-et-non-le-francais_a82075.html>.

LEIRIS, Michel (1939). L'Âge d'homme. Paris : Gallimard.

SOLLERS, Philippe (2006). Carnet de nuit. Paris : Gallimard.

\section{NOTES}

1. Clarisse Fabre rapporte que Nicolas Sarkozy a dit en 2006 qu' " un sadique ou un imbécile avait mis dans le programme d'interroger les concurrents sur La Princesse de Clèves », puis, qu'en 2008, il a ajouté : «Car ça vaut autant que de savoir par cœur La Princesse de Clèves. J'ai rien contre, mais... bon, j'avais beaucoup souffert sur elle» (Fabre, 2011). Voir < http://www.lemonde.fr/ cinema/article/2011/03/29/et-nicolas-sarkozy-fit-la-fortune-du-roman-de-mme-de-la-

fayette_1500132_3476.html > [disponible le 20/10/2015].

2. Le colloque intitulé "Crise : échec ou défi » de l'Asociación de Profesores de Francés de la Universidad Española a été tenu à Alcala de Henares du 26 au 28 mars 2014. La table ronde a été animée par M. Fidel Corcuera-Manso (U. Zaragoza), M. Julian Serrano Heras (U. Castilla La Mancha) et M. Joaquin Díaz-Corralejo Conde (U. Complutense).

3. L'idée est immédiatement traduite par l'objet qui la représente. Un smartphone par exemple est le résultat direct du mariage entre le désir de la maîtrise hystérique de la réalité (espace et temps inclus) et le génie de l'inventeur.

4. «Perversion » dans le sens barthésien du terme : toute dépense qui rend heureuse l'âme du consommateur, tout plaisir inconditionnel (voir Barthes, $1981: 249-250$ ).

5. Michel Leiris écrit : « Me tournant vers le torero, j'observe que pour lui également il y a règle qu'il ne peut pas enfreindre et authenticité, puisque la tragédie qu'il joue est une tragédie réelle, dans laquelle il verse le sang et risque sa propre peau. La question est de savoir si, dans de telles conditions, le rapport que j'établis entre son authenticité et la mienne ne repose pas sur un simple jeu de mots » (Leiris, 1939 : 17). Quant à la confrontation du moi dans l'acte d'écrire, elle émane, selon Annie Ernaux, de «(...) la nécessité que j'éprouve, comme Leiris, d'une corne de taureau, d'un danger dans l'exercice de l'écriture » (Ernaux, $2011: 102)$.

\section{RÉSUMÉS}

La modernité parle une nouvelle langue, celle du marché. Le statut de l'intellectuel privilégie la figure de l'économiste. Les sciences humaines en général et la littérature en particulier souffrent d'un glissement de terrain qui menace leur existence. Plusieurs pays ont déclaré la guerre à tout ce qui n'est pas science rentable. Dans cet article, nous essayerons de voir dans quelle mesure l'enseignement de la littérature relève du souci de l'humanité de demeurer humaniste au lieu de se contenter d'un pragmatisme qui réduit le citoyen à un simple objet de consommation.

Modernity speaks a market's language. The status of the intellectual favors the figure of the economist. The humanities (literature) suffer from a landslide that threatens their existence. Several countries have declared war on all that is not exact sciences. In this article we will try to 
see how literature protects humanism and rejects a pragmatic approach that reduces the citizen to a mere object of consumption.

INDEX

Mots-clés : littérature, Français, légitimité, enseignement, humanisme

Keywords : literature, French, legitimacy, education, humanism

\section{AUTEUR}

\section{SIDI OMAR AZEROUAL}

Un. Cadi Ayyad - Marrakech sidiomar.a[at] gmail.com 